\title{
LITERATURE ALERT
}

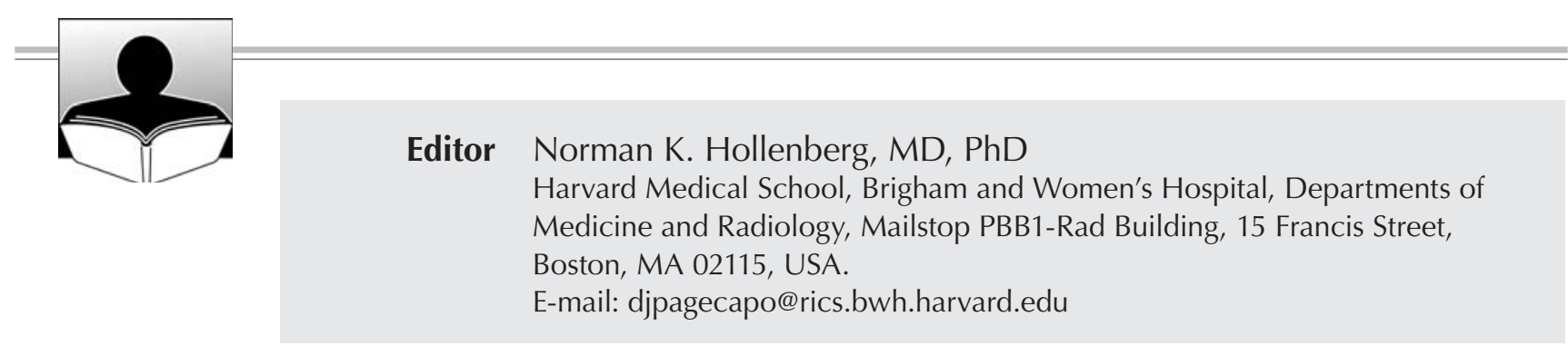

Whitmer RA, Karter AJ, Yaffe K, et al.: Hypoglycemic episodes and risk of dementia in older patients with type 2 diabetes mellitus. JAMA 2009, 301:1565-1572.

Abstract: Context: Although acute hypoglycemia may be associated with cognitive impairment in children with type 1 diabetes, no studies to date have evaluated whether hypoglycemia is a risk factor for dementia in older patients with type 2 diabetes.

Objective: To determine if hypoglycemic episodes severe enough to require hospitalization are associated with an increased risk of dementia in a population of older patients with type 2 diabetes followed up for 27 years.

Design, Setting, and Patients: A longitudinal cohort study from 1980-2007 of 16,667 patients with a mean age of 65 years and type 2 diabetes who are members of an integrated health care delivery system in northern California.

Main Outcome Measure: Hypoglycemic events from 1980-2002 were collected and reviewed using hospital discharge and emergency department diagnoses. Cohort members with no prior diagnoses of dementia, mild cognitive impairment, or general memory complaints as of January 1, 2003, were followed up for a dementia diagnosis through January 15, 2007. Dementia risk was examined using Cox proportional hazard regression models, adjusted for age, sex, race/ethnicity, education, body mass index, duration of diabetes, 7-year mean glycated hemoglobin, diabetes treatment, duration of insulin use, hyperlipidemia, hypertension, cardiovascular disease, stroke, transient cerebral ischemia, and end-stage renal disease.

Results: At least 1 episode of hypoglycemia was diagnosed in 1465 patients $(8.8 \%)$ and dementia was diagnosed in 1822 patients (11\%) during follow-up; 250 patients had both dementia and at least one episode of hypoglycemia (16.95\%). Compared with patients with no hypoglycemia, patients with single or multiple episodes had a graded increase in risk with fully adjusted hazard ratios (HRs): for one episode (HR, 1.26; 95\% CI, 1.10-1.49); two episodes (HR, 1.80; 95\% CI, 1.37-2.36); and three or more episodes (HR, 1.94; 95\% CI, 1.42-2.64). The attributable risk of dementia between individuals with and without a history of hypoglycemia was $2.39 \%$ per year $(95 \% \mathrm{CI}, 1.72 \%-3.01 \%)$. Results were not attenuated when medical utilization rates, length of health plan membership, or time since initial diabetes diagnosis were added to the model. When examining emergency department admissions for hypoglycemia for association with risk of dementia (535 episodes), results were similar (compared with patients with 0 episodes) with fully adjusted HRs: for 1 episode (HR, 1.42; 95\% CI, 1.12-1.78) and for 2 or more episodes (HR, 2.36; 95\% CI, 1.57-3.55).

Conclusions: Among older patients with type 2 diabetes, a history of severe hypoglycemic episodes was associated with a greater risk of dementia.

Editor's Comment: This is an important study. It addresses a common complication of type 2 diabetes mellitus in a very large patient population. The epidemiologic tools used are described in great detail, and the argument for their accuracy is persuasive. Clinically identified hypoglycemic episodes were associated with an increased frequency of dementia, and the more hypoglycemic episodes, the more likely that dementia would occur. As $11 \%$ of the patients in the study developed dementia, the issues are truly important.

When the United Kingdom Prospective Diabetes Study appeared more than a decade ago, one of the leaders in this field, Carl Erik Mogensen, wrote the accompanying editorial. He pointed out in that editorial that correction of hypertension had a much larger influence on natural history 
than did correction of the metabolic abnormality. This finding surprised Mogensen because, after all, diabetes mellitus is primarily a metabolic disease.

If tight metabolic control truly prevented complications, perhaps we would be prepared to accept an increased risk associated with hypoglycemia. But if the gain is marginal, then we should examine the cost very carefully. Inspection of Table 1 may provide an additional clue. Hypoglycemia occurred in $37.8 \%$ of those treated with insulin only, almost three times greater than the frequency in those who did not receive insulin.

This valuable database is likely to teach us many more important clinical lessons.

Disclosure: No potential conflict of interest relevant to this article was reported. 